\title{
Information and the Quantum World
}

\author{
Dennis Dieks
}

Received: 24 October 2015; Accepted: 8 January 2016; Published: 13 January 2016

Academic Editor: Jay Lawrence

History and Philosophy of Science, Utrecht University, P. O. Box 85170, 3508 AD Utrecht, The Netherlands; d.dieks@uu.nl; Tel.: +31-62-848-8914

\begin{abstract}
The concept of information is not different in quantum theory from its counterpart in classical physics: a sui generis quantum information concept is not needed. However, the quantum world is radically different from its classical counterpart. This difference in structure of the material world has important consequences for the amounts of information that can be stored in physical systems and for the possibilities of information transfer. In many cases, overlap between quantum states (non-orthogonality of states) blurs distinctions and impedes efficient information transfer. However, the other typical quantum feature, entanglement, makes new and seemingly mysterious ways of transporting information possible. In this article, we suggest an interpretational scheme of quantum mechanics in terms of perspectival physical properties that may provide an intelligible account of these novel quantum possibilities, while staying close to the mathematical formalism of quantum mechanics.
\end{abstract}

Keywords: perspectival quantum properties; relational properties; quantum information; entanglement

\section{Introduction: Quantum Information}

Quantum information has become a key term in the foundational literature of the last decades and the novel possibilities provided by it, for example quantum teleportation, have rightly attracted much attention. It is sometimes suggested that the concept of information itself has undergone a drastic meaning change due to these developments, and it has been proposed that quantum theory is about this conceptually novel type of information rather than about physical systems, or even that according to quantum theory nothing but quantum information exists in the world. It is less than clear, however, what the exact content should be of a new definition of information that could make such statements completely intelligible [1].

Fortunately, we do not need any such drastic conceptual step. Both in quantum physics and in classical physics, we can think of processes of information storage and information transfer as material processes in which messages are encoded in an "alphabet" furnished by states of physical systems and in which states of physical systems prepared at a "sender" are reproduced (not necessarily as states of the same systems) at the location of a "receiver". We refer to [1] for a comprehensive review and critical discussion of claims about the allegedly novel character of the concept itself of information in quantum theory, and an elaborate defense of the more traditional account of information along the lines just indicated.

When we take this traditional meaning of "information" in mind, it seems implausible-at least at first sight-that the prospects of efficient information storage and transfer are better in quantum theory than in classical physics. The reason is that, in quantum theory, states of physical systems, even if maximally complete (pure) and distinct, may "overlap": different quantum states need not be orthogonal. This stands in the way of unequivocally distinguishing between these states. Consequently, when a message, written in the letters of some alphabet, is encoded by translating its letters into different states of a quantum system, the receiver will face a problem that -in principle-is 
not present in classical physics. Consider, for example, a message written in binary code ("letters" 1 and 0 ). If 1 is encoded as $|p\rangle$ and 0 as $|q\rangle$, with $\langle p \mid q\rangle \neq 0$, decoding via measurements on the quantum states $|p\rangle$ and $|q\rangle$ will inevitably lead to a loss of information. By contrast to the case of ideal measurements on classical distinguishable states or on orthogonal quantum states, in which it is possible to completely retrieve the original message, performing yes-no measurements of the projectors $|p\rangle\langle p|$ and $|q\rangle\langle q|$ on a non-orthogonal pair of states $|p\rangle$ and $|q\rangle$, introduces a probability $|\langle p \mid q\rangle|^{2}$ of incorrect classification.

More precisely, for the special case in which the frequencies with which 1 and 0 occur in the original message are equal (and in the limit of very long messages), the following simple consideration determines the most efficient classification procedure. We should look for a projector $|x\rangle\langle x|$ such that the outcome 1 of its measurement can be interpreted as the letter 1 in the original message, and the outcome 0 as the letter 0 , with a minimal probability of error. The probability of correct classification in this case is $1 / 2|\langle x \mid p\rangle|^{2}+1 / 2\left(1-|\langle x \mid q\rangle|^{2}\right)$, so the value of this expression should be made maximal. From the geometrical relations in the two-dimensional space spanned by $|p\rangle$ and $|q\rangle$, we see that this is the case when $|x\rangle$ bisects the angle between $|p\rangle$ and $|q\rangle^{\perp}$ (the ket normal to $|q\rangle$ in the two-dimensional space; note that, in the real case, $1 / 2|\langle x \mid p\rangle|^{2}+1 / 2\left(1-|\langle x \mid q\rangle|^{2}\right)$ is half the sum of the squared cosines of the angles between $|x\rangle$ and $|p\rangle$ and $|q\rangle^{\perp}$, respectively). The associated optimum probability of correct classification (compare [2]) is $1 / 2\left\{1+\left(1-|\langle p \mid q\rangle|^{2}\right)^{1 / 2}\right\}$ (in the real case, this is $\cos ^{2}(\psi / 2)$, with $\psi$ the angle between $|p\rangle$ and $|q\rangle^{\perp}$ ). Thus, even when the most efficient method of decipherment is used, $N / 2(1-\cos \psi)$ of the total number of $N$ bits will be misread.

There is an alternative to the probabilistic classification just discussed: it is possible to perform more sophisticated measurements that enable a classification without error. However, in this approach, we have to accept that some measurement outcomes will not allow a classification at all. The best one can achieve by this procedure is that $N|\langle p \mid q\rangle|$ of the bits remain unclassified [2,3], so that, again, we are facing a loss of information.

This does not mean that messages sent via quantum coding will always remain partly illegible: one can devise strategies that make the probability of error as small as one wishes in the long run. A basic strategy here is to introduce redundancy by sending the same information multiple times: comparison of the measurement outcomes on repeated encoded words will make it possible to reconstruct the original message with an increasing level of reliability. Indeed, wrong classifications (or no-classification events) may be assumed to occur independently in repetitions, so that it will become increasingly improbable that mistakes will repeat themselves in exactly the same way. However, it is nevertheless clear that the best results that are attainable in this manner can be much more simply achieved in classical physics, or with orthogonal quantum states. Thus, from this vantage point, there is no reason to have recourse to the new possibilities afforded by quantum theory if one wants to improve on traditional methods of encoding and transmitting information.

The possible non-orthogonality of different quantum states has the further consequence, intimately related to the just-discussed blurring of classical distinctions, that statistical mixtures of quantum states cannot be uniquely decomposed into pure component states. An impure state $\rho$ that has been prepared by probabilistically mixing pure non-orthogonal states $|\alpha\rangle_{i}$, with associated probabilities $p_{i}, i=1, \ldots, N$, is empirically indistinguishable from a mixture of orthogonal pure states $|\lambda\rangle_{i}$ with probabilities $\lambda_{i}, i=1, \ldots, n$, where the $|\lambda\rangle_{i}$ and $\lambda_{i}$ are the eigenstates and eigenvalues, respectively, of the density operator $\rho\left(\rho=\sum_{i} \lambda_{i}\left|\lambda_{i}\right\rangle\left\langle\lambda_{i}\right|\right)$ and where $n$ denotes the number of dimensions of the system's Hilbert space. This equivalent mixture of orthogonal states behaves as a classical probability distribution, with probabilities $\lambda_{i}$, over distinct classical states. From Shannon's noiseless coding theorem [4], we know that the number of classical bits needed to transport a long random series of $N$ such states is asymptotically (i.e., $N \rightarrow \infty$ ) given by $N . H(\lambda)$, with $H(\lambda)$ the Shannon entropy of the probability distribution $\lambda, H(\lambda)=-\sum_{i} \lambda_{i} \log _{2} \lambda_{i}$. Thus, classically, we have to count with $2^{N . H(\lambda)}$ possible message sequences. In the quantum context, this translates into the 
following: to transmit the state $\rho^{N}$ with asymptotically perfect fidelity, we need a Hilbert space of $2^{N . H(\lambda)}$ dimensions. This means that the number of qubits (i.e., two-level quantum systems) needed so that their combined states can span this Hilbert space is given by $H(\lambda)$; this number of qubits is accordingly needed to transmit $\rho . H(\lambda)$ is the Shannon entropy of the classical probability distribution $\lambda_{i}$, but it is also equal to the Von Neumann entropy $S(\rho)$ of the mixed state $\rho, S(\rho)=-\operatorname{Tr}\left(\rho \log _{2} \rho\right)$. So instead of the standard result that the Shannon entropy quantifies the number of bits per state needed for ("almost", i.e., in the long run) undistorted transmission in a classical information channel, we obtain that the Von Neumann entropy specifies the number of qubits per state needed in a quantum channel. This noiseless quantum coding theorem was derived by Schumacher in his seminal paper on quantum coding [5].

It is clear from the above sketch of Schumacher's result that the concept of information that is presupposed here is the same as the classical one: we are dealing with messages coded in states of physical systems and their transport along a physical channel, with the aim of reproducing the original states as accurately as possible with a minimal amount of resources. However, the physical difference between classical states and quantum states, and between bits and qubits, is reflected in an essential difference in the possibilities of discrimination: in classical physics all different states can be distinguished-they are "orthogonal" in this sense-but in quantum theory we face the complication of non-orthogonality. This explains why the number of bits needed to transmit the information coded in a classical mixture is generally greater than the number of qubits needed for a quantum mixture: $S(\rho) \leq H(p)$. Indeed, the Shannon entropy of a probability distribution $\left\{p_{i}\right\}, i=1, \ldots, N$ can take the maximum value $\log _{2} N$, so that the "preparation entropy" connected with classically preparing a statistically mixed quantum state can grow indefinitely when the number of pure components of the mixed state increases. However, the Von Neumann entropy of the resulting $\rho$ can never become greater than $\log _{2} n$, with $n$ the number of dimensions of the space in which $\rho$ is defined. Again, what is responsible for this difference is that the non-orthogonality of the component quantum states washes out distinctions that in classical physics are in principle always accessible.

Thus, it seems that quantum messaging is generally less powerful than classical procedures of storing and forwarding information. We know, however, that this verdict is at best incomplete. Quantum information theory has grown into a large and successful new field of research, and for good reason: it does turn out to be possible to do better than what classical information transfer leads us to expect. The essential ingredient of these new possibilities is the use of quantum theory on a level beyond the just-described coding via the states of one quantum system, namely by employing entangled states involving two or more quantum systems.

It is well-known from other contexts, in particular discussions surrounding Bell inequalities, that the existence of entangled states in quantum theory is responsible for a feature of physical reality (loosely speaking: "holism" or "non-locality") that is mysterious from a classical point of view. It is this quantum trait of physical reality that is exploited in the new quantum information protocols.

Summing up, the success of quantum information theory is not due to a change in the meaning of information per se in the context of quantum theory but rather to the application of informational concepts to new and unexpected features of physical reality (the bearer of information) governed by quantum theory. In the examples that we briefly discussed above, the conclusion was that quantum encoding is generally less efficient than classical coding. However, when we turn to information transfer between parties that make use of shared entangled states, it will turn out that more can be done than is allowed by classical physics. This difference is due to the fact that the quantum world is differently structured from the classical world-it is exactly because the same concept of information transfer (viz. reproduction of sender states) is used both in the quantum and classical cases that a meaningful comparison is possible.

The purpose of the remainder of this article is to suggest a characterization of the relevant physical difference between the classical and the quantum worlds by means of a conceptual framework that makes use of perspectival (or relational) properties. The central idea here is to 
think of properties not as monadically belonging to a physical system but as defined from a "point of reference". This is an interpretational motif that has been around since the beginning days of quantum mechanics (see, e.g., [6], and, for a different recent proposal, [7]—see [8] for some remarks comparing [7] with the line taken here). The reason is that an interpretation of quantum mechanics in terms of perspectival properties accords well with the mathematical structure of quantum theory and appears to hold the promise of demystifying the notorious measurement problem, as we shall briefly illustrate in the following. More important for our purposes here, we think that a perspectival interpretational scheme may help to make it understandable why quantum processes of information transfer are in some respects more efficient than classical ones.

\section{Perspectives: Classical versus Quantum}

One might be tempted to consider perspectival properties even in the context of classical physics. For example, think of a situation in which an experiment is performed in a sealed room. An experimenter within the room will in this case become aware of a definite result, whereas someone on the outside may not be aware that an experiment has taken place at all [8]. In a philosophical mood, and perhaps inspired by empiricist thoughts, the outside observer might contemplate the conceptual possibility that there is no unique truth of the matter concerning whether there is a definite outcome or not but that this depends on the perspective taken: perhaps the outcome is definite from a perspective internal to the room, and not from an outside perspective.

Clearly, this description of the situation would not be in agreement with classical physics, nor with common sense. A perfectly consistent and satisfactory account can be given when one assumes that the outcome is definite tout court-that the outside observer just does not know what is the case. In classical physics, any indefiniteness of the sort we just discussed can be taken to be purely epistemic and without ontological significance.

Thus challenged, our external observer might undertake sophisticated experiments on the sealed room, to see whether it is possible to find traces of what has happened inside. (It may be remarked that the notion of a completely sealed room is somewhat problematic, because gravitational forces cannot be shielded off. In the context of the quantum version of the thought experiment, to be discussed in a moment, this remark may be of more fundamental significance than in the classical case because it shows that some degree of decoherence via gravity cannot be avoided. This observation may be important for the explanation of the classical limit of quantum mechanics. However, in the remainder of our argument, we shall make the usual assumption that effects of gravity may be neglected.) On the assumption that classical physics is right, we can predict that, if there is an experiment in which something is found at all, the evidence will be in agreement with the existence of a definite result of the experiment. Thus, there is no reason, within the framework of classical physics, to take seriously the unusual and extravagant notion of properties that are only defined relative to a perspective.

However, this argument depends on the empirical validity of classical physics. However, we know that classical physics is in fact insufficient to predict and explain what can be found in experiments: it should give way to quantum theory. Let us therefore look again at the just-described situation, but now from a quantum point of view. We shall base ourselves on unitary quantum theory (i.e., quantum theory with unitary time evolution, without collapses), as this is the simplest and most natural quantum mechanical scheme in agreement with the experimental data-a point to become clearer as we go on.

Unitary time evolution implies that once a measurement interaction inside the closed room has terminated and the internal experimenter has inspected the result, the state of the room's contents will be given by a linear superposition of terms. Each of these terms will be a product in which there is a state corresponding to a definite experimental outcome and an experimenter state representing awareness of that same outcome. Experiments of the last couple of decades have been yielding an ever growing amount of evidence confirming the correctness of such a description by means of a 
superposed state: experiments on so-called Schrödinger cat states have become almost routine, and their results strongly indicate that superpositions are indeed needed to do justice to the experimental facts (e.g., [9]). Accordingly, if our external observer wants to make correct predictions about the results of measurements of the sealed room, he had better use the full superposition. For example, if our experimenter is going to measure the projection operator $|\Psi\rangle\langle\Psi|$ (where $|\Psi\rangle$ stands for the superposed state of the room and its contents), the formalism tells us that he will find the result "1" with certainty. As already stated, predictions of this type are borne out by experiment.

Indications are therefore that we should accept that the room and its contents possess the property represented by $|\Psi\rangle\langle\Psi|$, instead of a property corresponding to one definite measurement outcome. That is, this must be the viewpoint taken by an external observer who can do experiments on the whole room and its contents. However, experience also tells us that, for an observer inside the room, exactly one definite result will materialize: for this internal observer, the correct description of the situation will correspond to this one definite property.

In other words, physical evidence plus the quantum formalism suggest that there are circumstances in which more than one description can be given of a physical situation. Of course, there is the threat of a contradiction here.However, this contradiction can be avoided if we relativize our descriptions, and the states assigned in these descriptions, to a perspective (internal and external perspectives in the above example). This suggestion leads to the proposal to assign perspectival or relational states: states of a physical system $A$ as defined from the perspective of a physical system $B$.

This idea has an important immediate merit: it makes it possible to reconcile unitary evolution during a quantum measurement interaction with the occurrence of definite outcomes. Indeed, the suggestion is that the properties associated with the superposition and the definite outcomes, respectively, relate to different perspectives: the superposition determines the properties assigned by an external observer who has not interacted with the system, the definite measurement outcomes belong to the perspective of the internal observer [6].

\section{Relational States and Properties}

A concrete scheme for defining relational states and properties was introduced in [10] and we shall briefly recapitulate it here. We do not propose this scheme as definitive or the only possible one but rather as a concrete illustration of how the idea of perspectival properties reflects features of the mathematical formalism of quantum theory. The question of how to deal with cases of degeneracy in this scheme has not been completely settled, and, in the sequel, (e.g., in the teleportation example of Section 6), we shall largely ignore possible complications relating to this point.

Our starting point is the usual Hilbert space formalism of quantum mechanics with unitary time evolution. This formalism is assumed to be applicable to all physical systems, so that there is no dividing line between a classical and a quantum realm-it is consequently natural to assume that there exists a quantum state of the whole universe. For the sake of simplicity, we shall take this universe state to be pure, $|\Psi\rangle$. We shall use this state, and other Hilbert space states to be introduced below, in order to assign physical properties to systems. The core idea here will be that Hermitian operators (observables) with vanishing dispersion in a state represent definite-valued physical quantities of the system in that state.

In order to proceed, we have to define states of physical systems $S$ that are proper parts of the universe $U$; here, we shall introduce our perspectivalism, although staying within the standard Hilbert formalism. We are going to introduce perspectival states $\rho_{R}^{S}$, which may be projector operators or density operators. The upper and lower index in $\rho_{R}^{S}$ reflect that the state of physical system $S$ will need the specification of a "reference system" $R$, with respect to which it is defined: $\rho_{R}^{S}$ is the state of $S$ with respect to $R$. As explained before, we want to make it possible that one and the same system, at one and the same instant of time, possesses different states and different properties with respect to different reference systems. 
The first case we shall consider is the one in which $R$ coincides with $S$ : the state of $S$ with respect to itself. This state we define as one of the projectors occurring in the spectral decomposition of the reduced density operator of $S$, i.e., the density operator that is obtained for $S$ by partial tracing over all degrees of freedom not pertaining to $S$ in $|\Psi\rangle\langle\Psi|$. If there is no degeneracy, this state will be a one-dimensional projector

$$
\rho_{S}^{S}=\left|\psi_{S}\right\rangle\left\langle\psi_{S}\right|
$$

or equivalently a vector state $\left|\psi_{S}\right\rangle$.

This particular way of assigning states and associated properties is in accordance with ideas of modal interpretations of quantum mechanics [11-16]. The assignment is meant to be probabilistic: which projector from the spectral decomposition of $S^{\prime}$ s reduced density operator is $\rho_{S}^{S}$ is not fixed by this property assignment. The theory only specifies probabilities (namely, the usual Born probabilities) for the various possibilities.

The state $\rho_{S}^{S}$, the "state of $S$ with respect to itself", fixes a set of well-defined physical properties for $S$, i.e., physical quantities that possess definite values: we take all observables that commute with $\rho_{S}^{S}$ as definite-valued. In the case in which the state is a vector $\left|\psi_{S}\right\rangle$, all operators of which $\left|\psi_{S}\right\rangle$ is an eigenvector possess the definite value given by the corresponding eigenvalue. For example, the observable $\left|\psi_{S}\right\rangle\left\langle\psi_{S}\right|=\rho_{S}^{S}$ possesses the definite value 1 . In the case of degeneracy, multi-dimensional projectors may be posited to be definite-valued, but we shall not go into the complications of the degenerated case. These properties derived from the state of $S$ with respect to itself are interpreted as properties possessed by $S$ "on its own", without reference to anything external.

The perspectival aspect enters for real when we consider states $\rho_{R}^{S}$ for systems $R$ that are outside $S$, so that $A \equiv U \backslash R$ contains $S$. By virtue of the Schmidt decomposition of $|\Psi\rangle\langle\Psi|$ (the state of the universe), there is a unique state of $A$ that is coupled to $\rho_{R}^{R}$ (in the sense of being perfectly correlated to it via $|\Psi\rangle$ 's Schmidt decomposition). This motivates the assumption that this correlated state of $A$ is the state of $A$ with respect to itself $\left(\rho_{A}^{A}\right)$ that is realized if $\rho_{R}^{R}$ is actual: States $\rho_{A}^{A}$ and $\rho_{R}^{R}$ come in correlated pairs. In view of the correlation with $\rho_{R}^{R}$ we also take this state $\rho_{A}^{A}$ to be the state of $A$ with respect to $R: \rho_{R}^{A}$. Now, since system $S$ is contained in $A$, the state $\rho_{R}^{S}$ can be defined as the density operator that follows from $\rho_{R}^{A}$ by taking the partial trace over the degrees of freedom in $A$ that do not pertain to $S$ :

$$
\rho_{R}^{S}=\operatorname{Tr}_{A \backslash S} \rho_{R}^{A}
$$

Any relational state of a system with respect to any other system outside of it can be determined by means of Equation (2).

For more about the dynamics of the states in this scheme (since most of the states follow by partial tracing from other states, states will generally not evolve unitarily), (joint) probabilities, the case of degeneracy and other details, see $[10,14,15]$.

\section{Quantum Perspectives}

Let us see what description results if we apply the scheme of the previous section to our thought experiment involving the sealed room. For the sake of simplicity, we replace that experiment by a universe $U$ consisting of three systems: an external observer $I$, a physical system "in the room" $D$ and an internal observer $F$ (we assume the $c_{i}$ to be different, so that there is no degeneracy). We take the initial state of this universe $|\Psi\rangle$ to be a product state: $|\Psi\rangle=\left|I_{0}\right\rangle \otimes\left|D_{0}\right\rangle \otimes\left|F_{0}\right\rangle$, with $\left|D_{0}\right\rangle=\sum c_{i}\left|D_{i}\right\rangle$, where the states $\left|D_{i}\right\rangle$ are eigenstates of the observable to be measured by the observer $F$. Using Von Neumann's scheme for unitary measurement interactions, we can represent the first stage of the experiment, in which $F$ performs a measurement in the room and notes its outcome, as follows:

$$
\left|I_{0}\right\rangle \otimes\left|D_{0}\right\rangle \otimes\left|F_{0}\right\rangle \longrightarrow\left|I_{0}\right\rangle \otimes \sum c_{i}\left|D_{i}\right\rangle \otimes\left|F_{i}\right\rangle
$$


When the external observer $I$ inspects what has happened in the room, by interacting with $D$, this can similarly be represented as:

$$
\left|I_{0}\right\rangle \otimes \sum c_{i}\left|D_{i}\right\rangle \otimes\left|F_{i}\right\rangle \longrightarrow \sum c_{i}\left|I_{i}\right\rangle \otimes\left|D_{i}\right\rangle \otimes\left|F_{i}\right\rangle,
$$

in which the states indexed by different values of $i$ are mutually orthogonal.

We now apply the rules of the previous section to Equations (3) and (4) in order to determine the properties of the various component systems at the different stages of the process. From the final state in Equation (3) we see that $\rho_{F}^{F}$ is one of the projectors $\left|F_{i}\right\rangle\left\langle F_{i}\right|$, so that $F$ has registered one definite result $i$. Similarly, $D$ by itself has acquired a definite property (possibility $i=k$ being realized with probability $\left|c_{k}\right|^{2}$ ). The state of $I$ is, as expected, as it was before the experiment started, since $I$ has not yet been involved in the measurement. The state of $D$ plus $F$ for I is given by $\sum c_{i}\left|D_{i}\right\rangle \otimes\left|F_{i}\right\rangle$, from which it follows that the state of $F$ for $I$ is the improper mixture $\sum\left|c_{i}\right|^{2}\left|F_{i}\right\rangle\left\langle F_{i}\right|$, and analogously for the state of $D$ with respect to $I$. If $I$ knows the state $\rho_{I}^{D \& F}$ (from repeated experiments), and knows quantum mechanics and the interpretational scheme we are discussing, he will be able to conclude that there will be certain internal properties of $D$ and $F$ relating to $\rho_{D}^{D}$ and $\rho_{F}^{F}$, with a correlation between them, even though these internal properties are not definite for $I$ himself. The fact that these internal properties are only definite-valued relative to $D$ and $F$ does not preclude $I$ from knowing that this is the case. Importantly, however, the correlation between the internal properties is definite for $I$, in the sense that the operator $\sum\left|D_{i} F_{i}\right\rangle\left\langle D_{i} F_{i}\right|$ commutes with $\rho_{I}^{D \& F}$ and so has a definite value (namely, 1).

Thus, the states of $D$ and $F$ are found to depend on the reference system with respect to which they are defined: they are different when defined with respect to $D$ and $F$ themselves from when taken with respect to $I$. This sharpens what was qualitatively discussed in the previous section: after the experiment in the room, there is a clear-cut outcome of the experiment for the internal observer, whereas for the external observer, the situation is still objectively undecided. The latter statement should not be interpreted as expressing a lack of knowledge about what really is the case, but as meaning that, with respect to $I$, the systems $D$ and $F$ ontologically do not possess well-defined properties corresponding to measurement outcomes. Indeed, with respect to $I, \sum\left|c_{i}\right|^{2}\left|D_{i}\right\rangle\left\langle D_{i}\right|$ is the state of $D$. When, as before, we take as our definiteness criterion that projectors $P$ are definite if $\operatorname{Tr} \rho P=\sum\left|c_{i}\right|^{2}\left\langle D_{i}|P| D_{i}\right\rangle=1$, we find that the projector $\sum\left|D_{i}\right\rangle\left\langle D_{i}\right|$ is definite but that the individual projectors $\left|D_{i}\right\rangle$ are not.

This changes when $I$ comes into interaction with $D$ and $F$. As a result of this new interaction, the total state becomes as represented by the right-hand side of Equation (4). At the end of this process, $I$ has recorded a definite result: his state with respect to himself has become one of the states $\left|I_{i}\right\rangle$. With respect to $I$, the states of $D$ and $F$ will now be given by the corresponding $\left|D_{i}\right\rangle \otimes\left|F_{i}\right\rangle$. It follows that, with respect to $I$, there is now a definite outcome of the experiment: $D$ shows a definite result and $F$ displays the corresponding correlated result. This is understandable: the already existing correlation between the internal properties of $D$ and $F$ has been carried over to $I$ 's perspective via the interaction between $I$ and $D$.

In this manner, we arrive at a description of the world in which all states and physical properties of physical systems are defined with respect to reference systems. These relational states are fixed by the quantum formalism and organized on its basis, in such a way that the ensuing hierarchy of physical perspectival properties reflects the mathematical structure of unitary quantum mechanics in Hilbert space.

\section{Holism and Locality}

The world according to quantum theory, in this interpretational scheme, is strikingly different from the classical world. These differences are responsible for unexpected empirical results, and this also holds for the possibilities of information transfer. A well-known and, in this context, 
highly relevant feature of the quantum formalism is the occurrence of a type of holism, in the sense that entangled states of composite systems are not derivable from the individual (mixed) states of their components. Our interpretational scheme translates this mathematical feature into a holism of physical properties: there will be properties of global systems that cannot be reduced to properties of their parts. This holism will manifest itself in some form of non-locality in situations in which the component systems are at a spatial distance from each other.

We can use the results of the previous section to further explain the situation and the perspectival take on it. As we have seen, the rules of Section 3 say that, after the initial stage of the experiment, the combined system of $D$ and $F$ is for $I$ in an entangled pure state, whereas the states, again for $I$, of the component systems are improper mixtures. The associated physical properties are represented by observables commuting with the projector on the entangled state in the case of the global system (this includes the correlation property $\left.\sum\left|D_{i} F_{i}\right\rangle\left\langle D_{i} F_{i}\right|\right)$, and by projectors like $\sum\left|D_{i}\right\rangle\left\langle D_{i}\right|$ for the component states. Therefore, from the perspective of $I$, the total system possesses physical properties (correlations) that cannot be understood as constructed from the properties of the partial systems: a manifestation of holism. From the point of view of $F$, however, there is no direct empirical sign of holism: the experiment has ended with a definite outcome, this has been recorded by $F$, and, for $F$, the total system is characterized by the conjunction of these properties.

If $I$ knows quantum mechanics, he will be able to derive from the total state of $D$ and $F$ what the possible internal properties of $D$ and $F$ are-he therefore can verify by a theoretical argument that these internal properties do not suffice to reconstruct the global properties of $D$ and $F$ that he is encountering. Thus, I from his global perspective "sees" the holistic features of the system, whereas the perspectives of $D$ and $F$ do not suggest holism.

An important question is in what sense the holism we have just identified leads to non-locality in cases in which the component systems are spatially separated. The answer is partly obvious: if the properties of a spatially extended system do not supervene on the properties of its individual localized parts, there is non-locality in exactly this meaning of the term. However, we can also consider another sense of the question, namely whether in such Einstein-Podolsky-Rosen-like situations there are instantaneous or faster-than-light influences traveling from one part of the system to another. It is this dynamical sense of non-locality that is often at stake in debates about the implications of violations of Bell inequalities.

The essential features of the Einstein-Podolsky-Rosen case are already present in the experiment of Section 4. When the experiment in the sealed room has finished, the state of $D$ plus $F$ is entangled, with the consequence that for $I$ there is no definite outcome. However, when $I$ enters the room and looks, and thus enters in interaction with what is inside, a definite outcome will be realized for $I$, as follows from Equation (4). There is no hint of dynamical non-locality in this process, because we may assume that the interaction between $I$ and $D$ is purely local. However, as a result of this same interaction between $I$ and $D$ the state of $F$ for $I$ also changes, namely into a state containing the information that $F$ has noted the same outcome as $I$ is observing now. This change of $F^{\prime}$ s state with respect to $I$ takes place without causal contact between $I$ and $F$. It may therefore appear that there is an influence on $F$ arising from the local interaction between $I$ and $D$. If we imagine a room that is very big, with $D$ and $F$ at a large distance from each other, such an influence should have to propagate superluminally.

However, attention to the relational character of the states takes away this impression of superluminal signalling. It is true that there is no physical interaction between $I$ and $F$, and that $I$ 's observation of $D$ cannot have a causal effect on $F$. However, I's own state is modified during the (purely local) interaction with $D$, and since we are considering the state of $F$ with respect to $I$, it need not cause bewilderment that this relational state becomes different from what it was. As a result of I's investigation of the properties of $D, I^{\prime}$ s state by itself becomes aligned with $D$ 's state by itself (cf. Equation (4)); it is therefore not strange that $F^{\prime}$ s state with respect to $I$ becomes the same as it already was with respect to $D$. We do not need to invoke non-local influences here: all changes can be 
understood from the combination of local physical interactions, the relational character of properties and states, and correlations that already existed independently of the interactions.

This line of reasoning carries over to the case of the Einstein-Podolsky-Rosen experiment (see [10]). Einstein, Podolsky and Rosen famously proposed that "if, without in any way disturbing a system, we can predict with certainty (i.e., with probability equal to unity) the value of a physical quantity, then there exists an element of physical reality corresponding to this physical quantity" [17]. We may accept this criterion, but within our relational framework its application does not lead to the conclusion that for $I$ the definite properties of $F$ were already there before the start of the experiment. The crux is that although there are no physical disturbances going from $I$ to $F, I^{\prime}$ s observation of $D$ does lead to a change, namely a change in I's perspective. This is relevant because of the relational, perspectival, character of the state we are considering: it is a state of $F$ with respect to $I$, and this makes the local change in I important.

The nature of quantum reality is thus quite different from that of classical reality according to this proposed scheme of a hierarchy of perspectives. Nevertheless, to some extent, quantum theory itself hides these revolutionary features from view. For example, when different observers compare their findings, they will agree on what they have seen, as illustrated by the agreement between $I$ and $F$ about the outcome of the experiment. This is a general feature of the formalism [10]. Furthermore, there are the omnipresent effects of decoherence that tend to wash out the effects of entanglement and therefore of differences in perspective. Still, in principle, the existence of superpositions (as in the case of the state of $D$ and $F$ before $I$ has taken a look at $D$ ) can be detected experimentally, and presently available experimental results strongly indicate the untenability of a fully classical picture.

The characterization of quantum reality by means of perspectival properties thus may hold the promise that a dynamically local account of what goes on in quantum processes is possible. As we have seen, the core idea is that physical measurement interactions are purely local but can nevertheless be responsible for the creation of new perspectives from which even far-away systems look different. We shall now investigate how this account fares in the case of quantum teleportation, one of the most dramatic examples of the new possibilities of information transfer allowed by quantum theory.

\section{Quantum Teleportation}

The quantum teleportation protocol makes use of a more sophisticated version of entangled states experiments than we have discussed in Section 5. In this new version, two experimenters, Alice and Bob, share an entangled state of two two-state particles (qubits), for example $|\Psi\rangle_{1}=$ $\frac{1}{\sqrt{2}}\left(|0\rangle_{a}|0\rangle_{b}+|1\rangle_{a}|1\rangle_{b}\right)$. The particle whose states are indexed by $a$ is close to Alice, so that she can perform experiments on it; and the $b$-indexed particle is close to Bob. In the sequel, it will be important that the state $|\Psi\rangle_{1}$ is the first of four mutually orthogonal, maximally entangled two-qubit states that are known as the "Bell states" $|\Psi\rangle_{j}, j=1,2,3,4$-these four states form the "Bell basis" of the four-dimensional Hilbert space of qubits $a$ and $b$.

The new element compared to our earlier examples is that now a third qubit $c$ arrives near Alice's position. This qubit is in a state $|\phi\rangle$, which need not be known to Alice. The total state of the three qubits, plus Alice and Bob (also described as quantum systems), is at this stage (cf. [18]):

$$
\left|A_{0}\right\rangle\left|B_{0}\right\rangle|\phi\rangle_{c}|\Psi\rangle_{1}
$$

with $\left|A_{0}\right\rangle$ and $\left|B_{0}\right\rangle$ the "neutral states" of Alice and Bob, respectively—that is, their states before any interaction between them and the system of the three qubits has taken place. This state can also be written as:

$$
\left|A_{0}\right\rangle\left|B_{0}\right\rangle \Sigma_{j}\left|\Psi^{a c}\right\rangle_{j} U_{j}|\phi\rangle_{b}
$$


in which the $\left|\Psi^{a c}\right\rangle_{j}$ states are the entangled Bell states of the qubits $a$ and $c$ (both located close to Alice), $U_{j}$ is a unitary operator that is independent of $|\phi\rangle$ and only acts on qubit $b$, and $|\phi\rangle_{b}$ indicates the counterpart of particle $c$ 's state in the Hilbert space of particle $b$ (the three single-qubit Hilbert spaces are of course isomorphic). The state (6) is the same as the state (5): to go from Equations (5) to (6) only simple algebraic manipulation is needed.

The teleportation process now proceeds as follows: Alice performs a measurement in the Bell basis, with non-degenerate eigenvalues, on her $a$ and $c$ qubits. As we see from Equation (6), she will find one of the results $j=1,2,3$ or 4 ; i.e., she will acquire two bits of information. This value of $j$ is sent to Bob via a classical channel, and Bob subsequently uses the value he receives to perform the unitary transformation $U_{j}^{-1}$ on the state of his qubit $b$. As a result, he has now at his disposal a qubit $b$ in the state $|\phi\rangle$, the same state in which qubit $c$ originally arrived near Alice. This state $|\phi\rangle$ has thus been "transported" from the vicinity of Alice to Bob; and this has been done by transmitting only two bits of information. This is remarkable: had Alice attempted to transport the state of $c$ by first determining this state (in very many repetitions of the situation) and then conveying the result to Bob, she would have needed many more bits since an arbitrary state has the form $|\phi\rangle=\alpha|0\rangle+\beta|1\rangle$ with $\alpha$ a complex number $\left(0 \leq|\alpha|^{2} \leq 1\right)$ that Alice would have to specify. (It would however, be a fallacy to state that Alice in teleporting $|\phi\rangle$ has actually succeeded in transmitting this vast amount of information: as rightly observed by Timpson (Section 4.3, [1]), the value of $\alpha$ is not available to Bob after the teleportation since he cannot determine $|\phi\rangle$ in a single run of the experiment. The amount of information that Bob can actually extract from $|\phi\rangle$ is only one bit, less than the two bits transmitted by Alice (Section 4.2.1, [1]).

The just-described steps in the teleportation procedure can be represented by a unitary transition from Equation (6) to:

$$
\Sigma_{j}\left|A_{j}\right\rangle\left|B_{j}\right\rangle\left|\Psi^{a c}\right\rangle_{j}|\phi\rangle_{b}
$$

In this equation, $\left|A_{j}\right\rangle$ and $\left|B_{j}\right\rangle$ stand for Alice's and Bob's states, respectively, after they have received the information $j$. It is clear from Equation (7) that $b$ will at the end find itself in state $|\phi\rangle$ whatever the outcome of Alice's measurement happened to be.

The central question raised by the teleportation protocol is how information about $|\phi\rangle$ is transported from Alice to Bob. It seems that this information cannot be contained in the two bits transmitted by Alice: these bits reveal the value of $j$ and thus merely lift the uncertainty about whether $1,2,3$ or 4 was measured by Alice. Further, it seems impossible that the shared entangled state in itself is able to transport the state of $c$. Indeed, if we follow the standard practice of determining a state for Bob's qubit $b$ by taking a partial trace in the total states (5) and (6), we find that both before and after Alice's measurement $b$ is in the uninformative mixed state represented by half the unity operator (defined in $b^{\prime}$ s two-dimensional qubit space). How could this maximally mixed state be transformed into the pure state $|\phi\rangle$ with the help of a mere two bits of information?

Different interpretations of quantum mechanics provide different accounts of how this could happen (Section 4.5, [1]). We believe that the scheme of perspectival properties set out in this article entails a worthwhile addition to this repertoire of explanations because of its direct correspondence with the mathematical formalism of unitary quantum theory (it is a rival, in this respect, to the many-worlds interpretation).

To see how the perspectival explanation works out, the first thing to notice is that Equation (6) looks similar to the states discussed in Section 3, so that we may apply analogous ideas. Concretely, even before Alice or Bob enter into interaction with any of the qubits, there exists a strict correlation-relative to both Alice and Bob-between the $a, c$ system and the $b$ qubit. Internally, from the perspective of the $a, c$ system, the state of $b$ relative to the state $\left|\Psi^{a c}\right\rangle_{j}$ is given by $U_{j}|\phi\rangle_{b}$, so that before the teleportation procedure starts from the viewpoint of $a, c$ the qubit $b$ already is "almost" in the state $|\phi\rangle$. (It is "almost" $|\phi\rangle$ in the sense that the state correlated to $\left|\Psi^{a c}\right\rangle_{j}$ is not $|\phi\rangle$ itself but is rotated over an angle that depends on $j$-to change this into a correlation with $|\phi\rangle$ itself, these 
rotations must be undone and this needs the transfer of the appropriate value of $j$, so two classical bits of information).

Now, when Alice performs her local measurement on $a$ and $c$, she aligns herself with the internal perspective of the $a, c$ system. She takes over the perspective associated with the state $\left|\Psi^{a c}\right\rangle_{j}$ and therefore becomes correlated with $U_{j}|\phi\rangle_{b}$ herself. At this stage, Bob is not yet part of the internal viewpoint so that for him everything is as before as far as the $(a, b, c)$-system is concerned. Moreover, with respect to Bob, there is no definite outcome of Alice's measurement: even Alice is in an uninformative mixture with regard to the value of $j$. This indefiniteness is ended by Bob's local interaction with the message containing the value of $j$ : this interaction (which essentially is a local interaction between Bob and Alice by means of which Bob inspects Alice's properties, like $I$ inspected $D$ in Section 4) puts Bob into the same perspective as Alice. The information given to Bob, the two bits contained in the value of $j$, tells him with which of the four possible $b$-states Alice already was correlated before the message arrived-and so with which of the four states he himself is now also correlated. This is sufficient for deciding which of the four rotations he has to apply to obtain $|\phi\rangle_{b}$.

This explanation of teleportation to some extent resembles familiar explanations from classical physics: an already existing correlation between systems near Alice and Bob, respectively, is exploited so that information about Alice's local state of affairs enables Bob to know what things are like on his side. That there are correlations between Alice's and Bob's regions is in itself not surprising because $a$ and $b$ were prepared in a correlated state. It is surprising however, from a classical viewpoint, that the inclusion of $c$ in the correlated qubit system $(a, b)$ leads to a correlated three-qubit state in which the initial state of $c$ near Alice is reflected in $b$ near Bob. This could not be understood if local properties of $a, b$ and $c$ were the whole story (as it necessarily must be the case in local classical accounts). In the quantum case, if we wish to discuss the $a, b, c$-system as a whole, we need a point of view that is external to this three-qubit system: we need global properties that are defined with respect to an external reference system. As we have seen in the example of the experiment in the sealed room, such global properties will not reduce to internal properties in cases in which the global state is entangled-this is the typical quantum holism. In the teleportation case, all combined $a, b, c$ properties with respect to Alice or Bob, before Alice and Bob have interacted with the qubit system, are given by $\Sigma_{j}\left|\Psi^{a c}\right\rangle_{j} U_{j}|\phi\rangle_{b}$, and not by the internal qubit properties. From this global description, it follows that, before any interactions, as a kinematical rather than dynamical effect, the three-qubit system is characterized by a strict correlation between $\left|\Psi^{a c}\right\rangle_{j}$ and $U_{j}|\phi\rangle_{b}$.

Summing up, the explanation of the success of the teleportation protocol consists of two components: first, the non-classical kinematical stratification of perspectives according to which composite systems may possess features that are not reducible to the properties of their components and, second, the exploitation of already existing correlations.

\section{Conclusions}

The concept of information is the same in quantum mechanics and classical mechanics: in both cases, information storage and transport consist in the coding of messages in alphabets defined by states of physical systems and their subsequent reproduction and decoding. However, the properties of quantum systems are vastly different from those of classical systems and this difference is responsible for discrepancies in the amounts of information that can be stored and transported. One essential distinctive feature compared to the classical world is that quantum states need not be orthogonal to each other; coding with such non-orthogonal states leads to less informative messages than classical coding. Of course, orthogonal states do exist in quantum theory: restriction to them brings us back to the classical situation. The classical distinguishability of different states is a special case of what we encounter in quantum theory.

The second important difference between the classical and quantum worlds is due to the occurrence of entangled states in quantum theory. Composite systems in such states possess irreducible global properties, not supervening on properties of their components. Such global 
properties may lead to the existence of correlations that are not understandable within a classical framework; and it turns out that such correlations can be cashed out by transferring information in ways that are classically impossible. The teleportation protocol illustrates this very well. Indeed, the qubit $b$ is in a maximally uninformative mixture with respect to both Alice and Bob at the beginning of the experiment, so it does not possess distinctive properties. Nevertheless, for Alice and Bob, there is a "correlation" property of the global three-qubit system that can be used to transmit information, namely a correlation between the states of $a, c$ on the one hand and $b$ on the other. Alice and Bob know before the start of their measurements that this correlation will correlate Alice to $b$ once a local measurement will have aligned Alice's perspective with that of the $a, c$ system. This is sufficient for them to be confident that the teleportation procedure will work.

The structure of this account follows the mathematical structure of quantum theory; but does it also provide us with an explanation of what happens in quantum teleportation? Evidently, this is not so from the viewpoint of classical physics: if we restrict our explanatory tools to the mechanisms recognized in classical physical theory, with local properties and causal influences propagating between them, we will not be able to achieve satisfactory explanations of what is going on in the quantum realm. However, it is an unjustified demand to restrict our "toolkit" of explanatory instruments this way: with the advance of science our models of explanation should also change and become more general (cf. [19])—it would be unscientific to require otherwise. Our intuitions are not absolutely rigid and can adapt to new theoretical insights, as is proved by the history of science. The classically bizarre correlations that exist according to quantum theory, and the difference between internal and external perspectives, may therefore well become incorporated into our resources for achieving intuitive explanation and understanding. We can certainly become accustomed to the kinematics of quantum systems, to the point of recognizing without explicit calculational verification the consequences of expressions like Equations (3) and (6). As soon as this insight is achieved, an intuitive understanding of how teleportation works is within reach.

Acknowledgments: It is a pleasure to acknowledge the 2015 Buenos Aires conference "What Is Quantum Information?" (organized by Olimpia Lombardi), which motivated and inspired this article.

Conflicts of Interest: The author declares no conflict of interest.

\section{References}

1. Timpson, C.G. Quantum Information Theory and the Foundations of Quantum Mechanics; Clarendon Press: Oxford, UK, 2013.

2. Jaeger, G; Shimony, A. Optimal Distinction between Two Non-Orthogonal Quantum States. Phys. Lett. A 1995, 197, 83-87.

3. Dieks, D. Overlap and Distinguishability of Quantum States. Phys. Lett. A 1988, 126, 303-306.

4. Shannon, C.E. The Mathematical Theory of Communication. Bell Syst. Tech. J. 1948, 27, 379-423, 623-656.

5. Schumacher, B. Quantum Coding. Phys. Rev. 1995, 51, 2738-2747.

6. London, F.; Bauer, E. La Théorie de l'Observation en Méchanique Quantique; Hermann \& Cie: Paris, France, 1939. (In French)

7. Rovelli, C. Relational Quantum Mechanics. Int. J. Theor. Phys. 1996, 35, 1637-1678.

8. Dieks, D. Objectivity in Perspective: Relationism in the Interpretation of Quantum Mechanics. Found. Phys. 2009, 39, 760-775.

9. Cavalcanti, E.G.; Reid, M.D. Criteria for Generalized Macroscopic and Mesoscopic Quantum Coherence. Phys. Rev. A 2008, 77, 062108.

10. Bene, G.; Dieks, D. A Perspectival Version of the Modal Interpretation of Quantum Mechanics and the Origin of Macroscopic Behavior. Found. Phys. 2002, 32, 645-671.

11. Bub, J. Interpreting the Quantum World; Cambridge University Press: Cambridge, UK, 1997.

12. Dieks, D. Resolution of the Measurement Problem through Decoherence of the Quantum State. Phys. Lett. A 1989, 142, 439-446.

13. Dieks, D.; Vermaas, P.E. (Eds.) The Modal Interpretation of Quantum Mechanics; Kluwer Academic Publishers: Dordrecht, The Netherlands, 1998. 
14. Dieks, D. Probability in Modal Interpretation of Quantum Mechanics. Stud. Hist. Philos. Mod. Phys. 2007, 38, 292-310.

15. Lombardi, O.; Dieks, D. Modal Interpretations of Quantum Mechanics. In The Stanford Encyclopedia of Philosophy; Spring 2014 Editon; Zalta, E.N., Ed.; Metaphysics Research Lab of Stanford University: Stanford, CA, USA, 2014

16. Vermaas, P.E.; Dieks, D. The Modal Interpretation of Quantum Mechanics and its Generalization to Density Operators. Found. Phys. 1995, 25, 145-158.

17. Einstein, A.; Rosen, N.; Podolsky, B. Can Quantum-Mechanical Description of Physical Reality be Considered Complete? Phys. Rev. 1935, 47, 777-780.

18. Braunstein, S.L. Quantum Teleportation without Irreversible Detection. Phys. Rev. A 1996, 53, $1900-1902$.

19. De Regt, H.W.; Dieks, D. A Contextual Approach to Scientific Understanding. Synthese 2005, 144, 137-170.

(C) 2016 by the author; licensee MDPI, Basel, Switzerland. This article is an open access article distributed under the terms and conditions of the Creative Commons by Attribution (CC-BY) license (http:/ / creativecommons.org/licenses/by/4.0/). 